\title{
Quantum chemical analysis of potential anti-Parkinson agents
}

\author{
NIMA RAZZAGHI-ASL ${ }^{\mathrm{a}, \mathrm{b}, *}$, SARA SHAHABIPOUR $^{\mathrm{a}}$, AHMAD EBADI $^{\mathrm{c}}$ \\ and AZAM BAGHERI ${ }^{\mathrm{C}}$ \\ a Department of Medicinal Chemistry, School of Pharmacy, Ardabil University of Medical Sciences, \\ Ardabil, Iran, PO code: 5618953141 \\ ${ }^{b}$ Drug and Advanced Sciences Research Center, School of Pharmacy, Ardabil University of Medical \\ Sciences, Ardabil, Iran \\ ${ }^{\mathrm{c}}$ Medicinal and Natural Products Chemistry Research Center, Shiraz University of Medical Sciences, \\ Shiraz, Iran \\ e-mail: razzaghinima@gmail.com; n.razzaghi@arums.ac.ir
}

MS received 7 August 2014; revised 18 March 2015; accepted 25 March 2015

\begin{abstract}
Monoamine oxidases (MAOs) are amine oxidoreductase falvoenzymes that belong to the integral proteins of the outer mitochondrial membrane. MAO exists in two distinct isoforms; MAO-A and MAO-B. Inhibition of MAO-A and MAO-B is important for developing antidepressant and antiparkinson agents, respectively. In the light of the above explanations, detailed structure binding relationship studies on the intermolecular binding components of MAO-B complexes may unravel the way toward developing novel anti-Parkinson agents. In the present contribution, intermolecular binding pattern for a series of experimentally validated 3-arylcoumarin MAO-B inhibitors (1-9) have been elucidated via molecular docking and density functional theory (DFT) calculations. Intermolecular binding energy components could not be analyzed by docking and due to this limitation, quantum mechanical $(\mathrm{QM})$ calculations including functional B3LYP in association with split valence basis set (Def2-SVP) were applied to estimate the ligand-residue binding energies in the MAO$\mathrm{B}$ active site. Moreover; results were interpreted in terms of calculated polarization effects that were induced by individual amino acids of the MAO-B active site. The results of the present study provide an approach to pharmacophore-based modification within the 3 -arylcoumarin scaffold for potent MAO-B inhibitors.
\end{abstract}

Keywords. MAO-B; Coumarin; Molecular docking; Quantum mechanical calculation.

\section{Introduction}

Monoamine oxidases (MAOs) are amine oxidoreductase falvoenzymes that belong to the integral proteins of the outer mitochondrial membrane. ${ }^{1}$ MAOs play a vital role in the metabolism of important amines in the central nervous system and peripheral tissues. ${ }^{2}$ Owing to the important role of MAOs in the metabolism of monoamine neurotransmitters, MAO inhibitors are used in the treatment of diverse neuropsychiatric and neurological disorders. ${ }^{3,4}$

In mammals, MAO exists in two distinctive isoforms that are identified as MAO-A and MAO-B. These two isoforms differ in their amino acid sequence, substrate specificity and sensitivity to selective inhibitors. ${ }^{5}$ Selective MAO-A inhibitors such as clorgyline are effective antidepressant drugs, while selective MAO-B inhibitors have been exploited as anti-Parkinson agents. ${ }^{6,7}$

MAO-B catalyzes the major catabolic pathway (oxidative deamination) of $\beta$-phenylethylamines such

\footnotetext{
*For correspondence
}

as dopamine in the brain. MAO-B inhibitors boost the dopaminergic neurotransmission as they prolong the activity of dopamine in the brain and hence have been used in the treatment of Parkinson's disease. ${ }^{8}$ Selective MAO-B inhibitors such as Selegiline and Rasagiline have been applied as potential disease-modifying agents in experimental clinical practice. It is important to know that selective MAO-B inhibitors may alleviate some symptoms of the Parkinson's disease such as resting tremor but not have the therapeutic effect at all. ${ }^{9}$ Considering this limitation, there is an urgent need toward novel MAO-B inhibitors with less side effects and higher potency.

Computer-aided drug discovery (CADD) is a rational design technique with important beneficial features. Proper application of CADD might lead to the reduced time, energy and expenditure toward novel therapeutic agents. CADD techniques are generally divided into two major categories; ligand-based and receptorbased methods. To run a receptor-based technique, 3D structure of the macromolecular target should be in hand. In this regard, available X-ray crystallographic 
structures of MAO (www.rcsb.org) provide an opportunity to elucidate the mechanisms of interactions between MAO and MAO inhibitors (MAOIs). Moreover; exploring the ligand-receptor interactions unravel the pharmacophoric requirements for designing potent and selective MAOIs with therapeutic applications.

Coumarins are chemically classified as benzopyrone derivatives with wide distribution in nature and biologically interesting properties. ${ }^{10}$ Theses potentialities introduced coumarins as privileged medicinal scaffolds for drug design aims. In particular it has recently been reported by several studies that 3 -substituted coumarins possess MAO-B inhibitory activity. ${ }^{11,12}$ A few reports on docking study of 3-substituted coumarins may be found in the literature. ${ }^{13,14}$ To provide additional insights into the binding mode, molecular docking studies of 3-phenylcoumarins (1-9) (scheme 1) ${ }^{5,15}$ were performed in the active site of MAO-B. Given the imperfection of docking simulations in providing the information on intermolecular binding energy components, ${ }^{16}$ subsequent to the docking studies, we estimated the participation of each amino acid in total binding energy via an

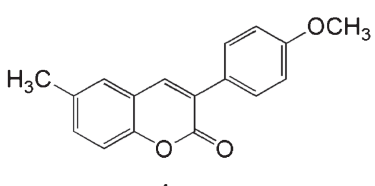

1<smiles>COc1ccccc1-c1cc2cc(C)ccc2oc1=O</smiles>

3<smiles>Cc1cc(Br)c2oc(=O)c(-c3ccccc3)cc2c1</smiles>

5<smiles>COc1cc(OC)cc(-c2cc3cc(C)cc(Br)c3oc2=O)c1</smiles>

7

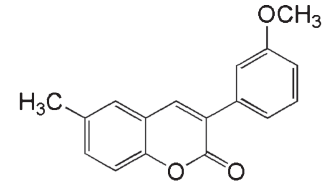

2

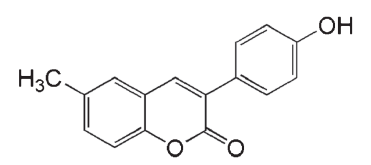

4
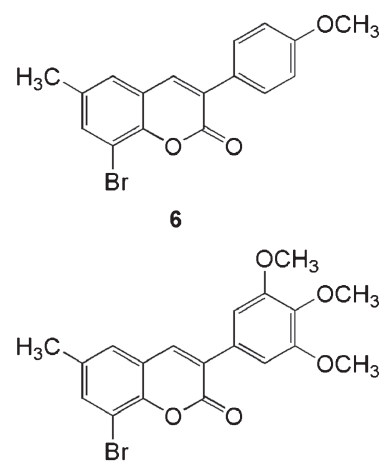

8

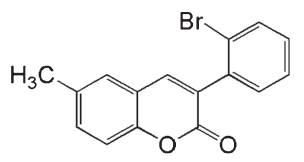

9

Scheme 1. Chemical structures of 3-phenylcoumarins under study (1-9). amino acid decomposition analysis through a quantum mechanical approach. In our opinion, such calculations are of significant importance in pharmacophore discernment and development within specific chemical scaffolds.

\section{Computational}

\subsection{Molecular docking}

Chemical structures of studied 3-phenylcoumarins were taken from literature. ${ }^{5,15}$ Flexible-ligand docking simulations were performed with AutoDock version 4.2. ${ }^{17} \mathrm{X}$-ray crystallographic structure of MAO-B was retrieved from the Brookhaven protein data bank (1S2Q; http://www.rcsb.org/). For preparation of a target protein, crystallographic ligand (Rasagiline) and water molecules were all removed from the original structure.

All the pre-processing steps for MAO-B file were performed via WHAT IF server (http://swift.cmbi.ru.nl/ servers/html/prepdock.html/European Molecular Laboratory Heidelberg, Germany) and AutoDock Tools 1.5.4 program (ADT). ${ }^{17,18}$ ADT program was used to merge non-polar hydrogens into related carbon atoms of the receptor and Kollman charges were also assigned. For docked ligands, non-polar hydrogens were added; Gasteiger charges assigned and torsions degrees of freedom were also considered by ADT program. Lamarckian genetic algorithm (LGA) was applied to model the interaction/binding between 3-phenylcoumarins and MAO-B active site. On the basis of validation study (represented in section 3), 100 GA runs; 27000 maximum generations; a gene mutation rate of 0.02 ; and a crossover rate of 0.8 were used for LGA method.

MAO-B was characterized by grid maps in the actual docking procedure. The grids were calculated using AutoGrid module (part of the AutoDock package). The grid included a map for each atom type in the ligand and also a map for electrostatic interactions. A size of grid was $60 \times 60 \times 60 \AA^{3}$ (distributed in the $\mathrm{x}, \mathrm{y}$, and $\mathrm{z}$ directions) and it was centered on the center of mass of the catalytic site of MAO-B with a spacing of $0.375 \AA$. Cluster analysis was performed on the docked results with regard to RMS tolerance of $2 \AA$.

\subsection{Ab initio studies}

The contribution of individual amino acid-ligand interaction energies in total binding energy were estimated using amino acid decomposition analysis (ADA). Amino acids (residues) involved in binding of 3-phenylcoumarin ligands to the MAO-B active site were chosen on 
the basis of previous reports on human MAO-B. ${ }^{19}$ For this purpose, all participating amino acids (Tyr60, Phe168, Leu171, Cys172, Tyr188, Ile198, Ile199, Gln206, Tyr326, Phe343, Tyr398 and Tyr435) along with the flavin cofactor (flavin adenine dinucleotide: FAD) were considered in their real electronic state. To mimic the original electron density, all the residues under study were truncated in their $\mathrm{C}$ and $\mathrm{N}$ terminals. $\mathrm{N}$-terminals were acetylated and $\mathrm{C}$-terminals were methyl amidated. All conformational and configurational features were held as original X-ray structure.

One of the restrictions in such modeling studies is the uncertainty of hydrogen positions in a typical Xray crystallographic file, and due to this limitation, we further optimized the heavy atom hydrogen bonds by B3LYP/ Def2-SVP method using heavy atom fixing (HAF) approximation (constrained optimizations). ${ }^{20}$ All the interaction energies were estimated by the same method and basis set. In energy calculations, longrange dielectric effects of protein were considered using COSMO model..$^{21}$ The full calculations were done with the ORCA quantum chemistry package. ${ }^{22}$

\section{Results and Discussion}

\subsection{Molecular docking}

Modern drug discovery strategies are dependent on crystallographic 3D structural information of the relevant biomolecular targets. Available X-ray crystallographic data on Protein Data Bank facilitated the structure based design of various enzyme inhibitors. In this regard, docking simulation is a key tool in structural molecular biology and computer-assisted drug design (CADD). To explain more, the stereoelectronic fit of ligand and receptor via lowest energy pathway may be checked by docking simulations. ${ }^{23}$ Numerous successful applications of molecular docking in drug discovery efforts have been reported. ${ }^{24,25}$ One of the most popular docking packages is AutoDock. Literature review shows that AutoDock has offered several fruitful advantages in the field of drug design (The AutoDock website. http://autodock.scripps.edu) ${ }^{26}$

3.1.1 Docking validation step: To predict predominant binding mode of a cognate (co-crystallographic) ligand, ${ }^{27}$ the structure of a cognate ligand was extracted and re-docked into its receptor (self-docking). Success is described in terms of comparison of root mean square deviation (RMSD) of the Cartesian coordinates of the atoms of the ligand in the docked and crystallographic conformations. A PDB-derived MAO-B structure including Rasagiline as cognate ligand (1S2Q) was subjected to docking validation procedure. Selection of the appropriate MAO-B structure was done on the basis of crystallographic resolution and lack of missing residues (http://www.rcsb.org/).

Regarding RMSD values and also conformation population in the top-ranked cluster of AutoDock output file, we decided to run all of the docking simulations on 1S2Q structure as a validated crystallographic target for MAO-B (table 1).

3.1.2 Binding mode and binding affinities: 3-phenylcoumarin structures (1-9) were all successfully docked into the active site of MAO-B. Docking outputs were all supported by high cluster populations. The results of the docking simulation are summarized in table 2. For comparison, experimental inhibition constants were also incorporated into table 2. All the MAO-B inhibitory activities were retrieved from the in vitro measurement of the enzymatic activity of human recombinant MAO isoforms in BTI insect cells infected with baculovirus. ${ }^{5,15}$

All the molecules under study shared a common structural motif of 6-methylcoumarin while compounds 5-8 possessed a bromine substituent on the $\mathrm{C} 8$ position of coumarin ring. Bearing this in mind, candidate molecules were evaluated within two distinct groups (C8-halogenated and C8-nonsubstituted) and for each

Table 1. Docking validation results for Rasagiline/MAO-B complex using AutoDock4.2.

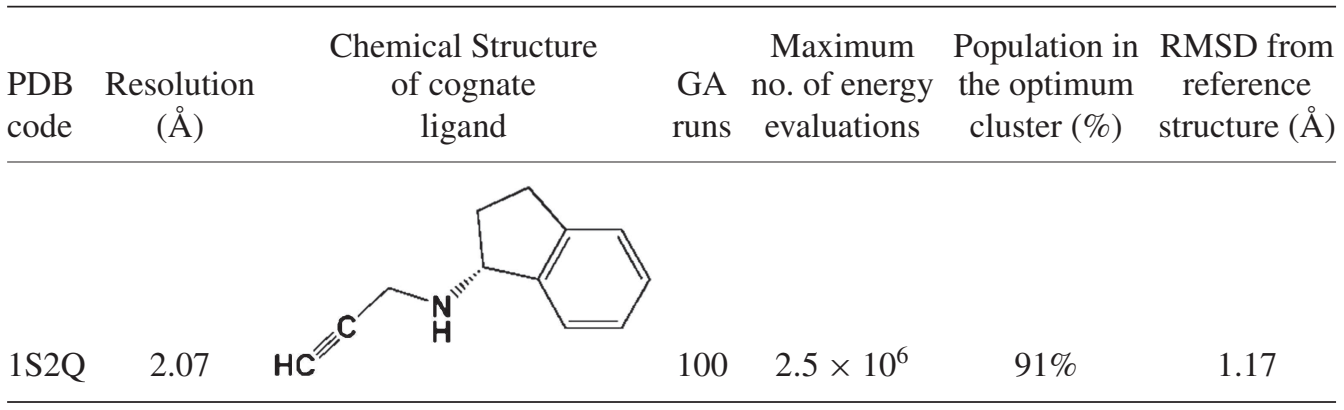


Table 2. Docking simulations results for 3-phenylcoumarins in the active site of MAO-B.

\begin{tabular}{lccc}
\hline & & \multicolumn{2}{c}{ AutoDock binding affinities (kcal/mol) } \\
\cline { 3 - 4 } Comp. & $\mathrm{pIC}_{50}{ }^{\mathrm{a}}$ & $\begin{array}{c}\text { Final docked } \\
\text { energy }\end{array}$ & $\begin{array}{c}\text { Estimated Free } \\
\text { Energy of Binding }\end{array}$ \\
\hline 1 & 7.884 & -9.69 & -9.09 \\
2 & 9.097 & -10.15 & -9.56 \\
3 & Inactive & -9.63 & -9.03 \\
4 & 6.808 & -9.44 & -8.85 \\
5 & 7.957 & -9.70 & -9.40 \\
6 & 8.491 & -10.26 & -9.67 \\
7 & 8.148 & -10.96 & -10.07 \\
8 & 5.311 & -11.03 & -9.83 \\
9 & 5.367 & -10.08 & -9.78 \\
\hline
\end{tabular}

${ }^{\mathrm{a}} \mathrm{All}$ pIC $\mathrm{C}_{50}$ values were calculated from the reported $\mathrm{IC}_{50} \mathrm{~s}$ in references. ${ }^{5,11}$

subclass, a prototype docked pose in the binding pocket of MAO-B was explored (figures 1-3). Figure 3 represents a binding mode of 3-(2'-bromophenyl)-6-methylcumarin (9) in the active site of MAO-B. For more clarification, obtained binding poses of 3-phenylcoumarins were compared with that of Rasagiline (figures 1-3).

The indan ring of Rasagiline is perpendicular to the benzopteridinedione ring of FAD with the nearest position to the central pyrazine ring. Detailed interaction features of Rasagiline in the binding pocket of MAO$\mathrm{B}$ may be found elsewhere. ${ }^{28}$ Careful inspection of the active site indicated that major binding contributions of the inhibitors were lipophilic contacts via hydrophobic residues of MAO-B. Similar results were also reported for a series of 3-heteroarylcoumarin derivatives. ${ }^{13}$ It should be emphasized that hydrophobic interactions are composite in nature and their careful analysis in a single model would face some inadequacies, but from the least point of view, some aspects on active site oriented pose of the ligand may be recognized via molecular modeling approaches.

All 3-phenylcoumarins participated in H-bonding with Cys172 via their coumarin carbonyl oxygen. The coumarin ring in the docked molecules was oriented a little closer to the Ile198, Ile199 and Cys172 when compared to Rasagiline. This new direction provided H-bonding with Cys172. 3-phenyl substituent of coumarin ring in 1-9 was located between Tyr326 and Ile199 residues making a $\pi-\pi$ stacking interactions with Tyr326.

Pursuing the activity profile of compounds 1-4 (table 2), it was revealed that para-methoxy (1) and meta-methoxy (2) groups on 3-phenyl moiety of coumarin ring could be better tolerated than ortho-methoxy groups (3). In this case, different observed activities might be attributed to the possible steric clash between C2'-substituted groups and Gln206. Such steric clashes could be envisaged for weak MAO-B inhibitor 9 due to the additional meta-substituted methoxy group. Moreover; superior activity of meta-methoxy substituted

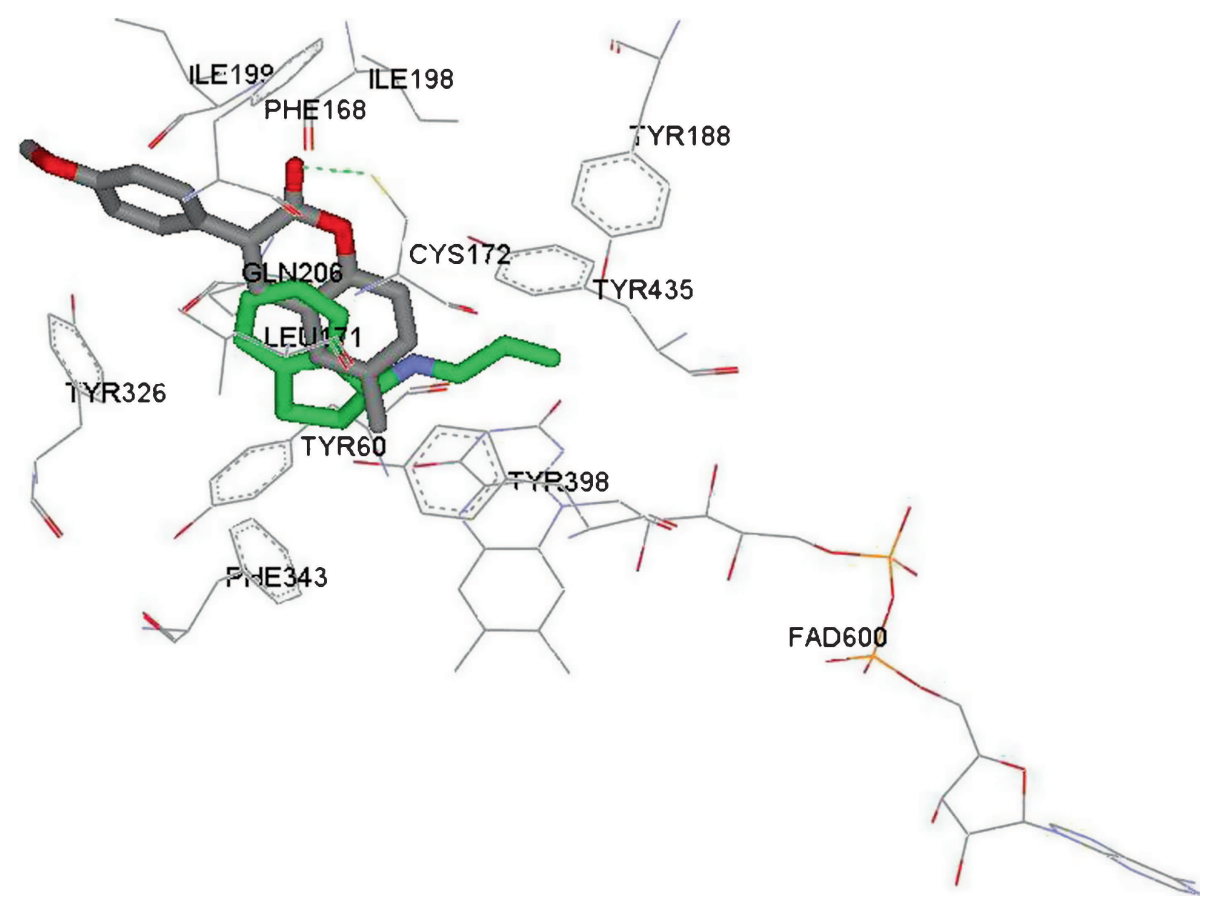

Figure 1. Docking result of 3-(4'-Methoxyphenyl)-6-methylcumarin 1 (orange stick model) with MAO-B (Rasagiline binding pose is presented by green stick model). 


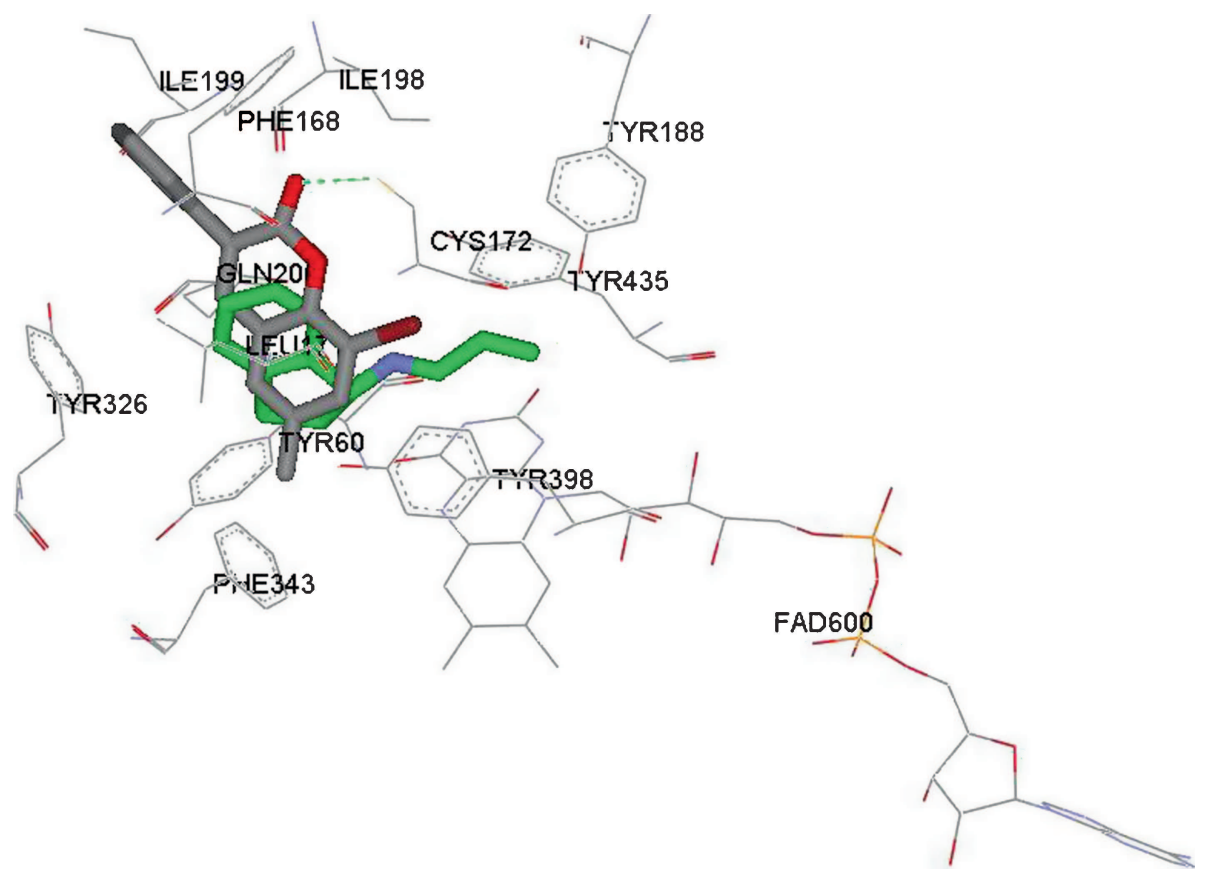

Figure 2. Docking result of 8-bromo-3-phenyl-6-methylcumarin 5 (orange stick model) with MAO-B (Rasagiline binding pose is presented by green stick model).

compound (2) could be the result of orientation towards Phe168 residue and occupying this lipophilic cavity. Similar trends might be presumed for the lower MAO-B inhibitory activity of compound $\mathbf{9}$ (figure 3 ).

Lower MAO-B inhibitory activity of $\mathbf{4}$ indicated an inappropriate orientation of para-hydroxy group in the active site of MAO-B and in confirmation of this, our obtained results showed no sign of $\mathrm{H}$-bonding between para-hydroxy moiety and MAO-B active site. None of the modelled molecules were adequately close toward Tyr435 and Tyr188 to make efficient lipophilic interactions except compounds 5, 6, 7 and 8 bearing a

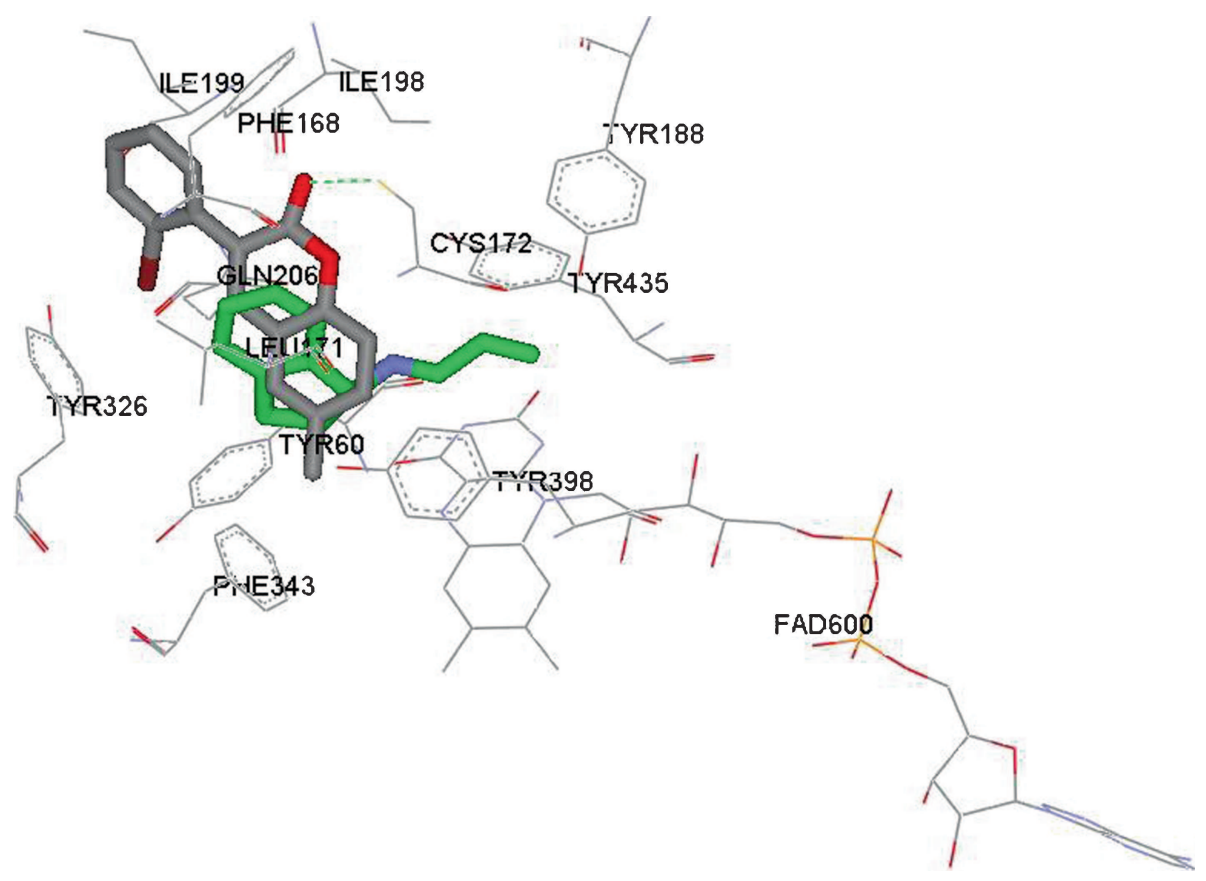

Figure 3. Docking result of 3-(2'-bromophenyl)-6-methylcumarin 9 (orange stick model) with MAO-B (Rasagiline binding pose is presented by green stick model). 
bromine substituent on their $\mathrm{C} 8$ position of coumarin ring (figure 2).

3.1.3 Regression analysis of docking affinities: None of the AutoDock binding affinities (Estimated free energy of binding and final intermolecular energy) exhibited correlation with in vitro MAO-B inhibitory activities. But a further regression analysis on the basis of modified AutoDock binding energies (considering a number of conformation clusters and top-ranked population in the AutoDock output file) led to enhanced correlation with biological data (figure 4). To get the detailed information on the proposed AutoDock binding energies, readers are referred to our previous report in the field. ${ }^{29}$ In our opinion, modified AutoDock affinities may provide a more rational design of MAO-B inhibitors.

\subsection{QM based binding energy analysis}

Molecular docking is a well-established method to achieve ligand-receptor binding patterns. In spite of this feature, no information on individual ligand-amino acid binding energies could be acquired upon docking. Given this, we were convinced to estimate the binding energies of a prototype 3-phenylcoumarin derivative with individual residues of the MAO-B binding site. Similar binding energies for the complex of RasagilineMAO-B were also calculated.

8-bromo-3-(3', 5'-dimethoxyphenyl)-6-methylcumarin (7) was selected as a model molecule for our QM analysis since it possessed diverse substitution pattern along with high in vitro MAO-B inhibitory activity $\left(\mathrm{IC}_{50}=7.12 \mathrm{nM}\right) .{ }^{15} \mathrm{~A}$ model system including twelve amino acids of the MAO-B active site (Tyr60, Phe168, Leu171, Cys172, Tyr188, Ile198, Ile199, Gln206, Tyr326, Phe343, Tyr398 and Tyr435) plus the FAD

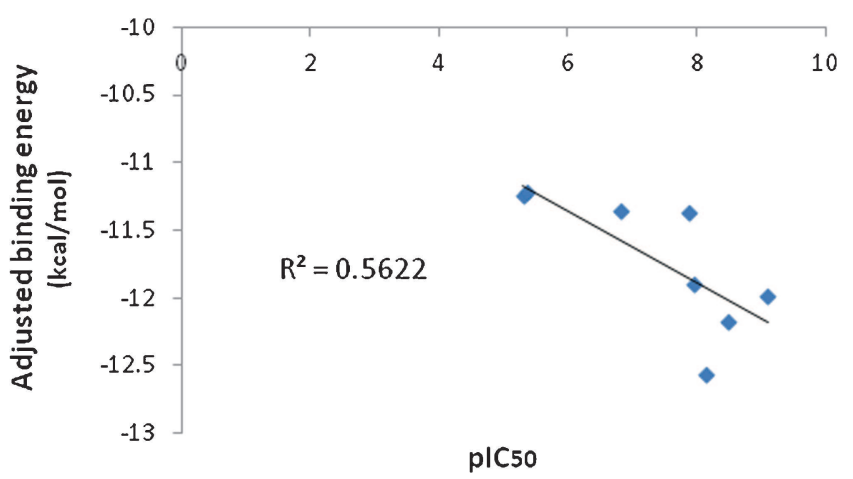

Figure 4. Plot of modified AutoDock MAO-B binding affinities versus in vitro MAO-B inhibitory activities for studied 3-phenylcoumarin derivatives (1-9). cofactor was constructed. Ligand-residue binding energies $\left(\Delta \mathrm{E}_{\mathrm{b}}\right)$ can be calculated by the Eq. (1):

$$
\Delta E_{b}=E_{L R}-E_{R}-E_{L}
$$

In the above equation, $\mathrm{E}_{\mathrm{LR}}$ is ligand-residue interaction energy, while $E_{R}$ and $E_{L}$ stand for the electronic energies of unbound residues and ligand, respectively. Binding energies of compound 7 and Rasagiline with individual amino acid residues comprising the MAO-B binding site at the B3LYP/ Def2-SVP level of calculation are summarized in figures $5 \mathrm{a}$ and $5 \mathrm{~b}$, respectively. It should be noted that all the calculations of ligand-receptor energies were performed in the gas phase without the involvement of desolvation energy in the binding energy.

For compound 7, Cys172, Ile198 and Tyr398 were the residues participated in $\mathrm{H}$-bonding $(-2.56 \mathrm{kcal} / \mathrm{mol})$ and lipophilic contacts $(-2.56$ and $-1.72 \mathrm{kcal} / \mathrm{mol})$ to the ligand, respectively. One important point is that the estimated binding energy for Cys172 might not be exclusively translated into $\mathrm{H}$-bond interaction with Cys172 since sulfur is a weaker H-bond donoracceptor than oxygen or nitrogen. Moreover; our binding maps showed that the close orientation of Cys172 and compound $\mathbf{7}$ in the active site of MAO-B provided more electrostatic interactions between compound 7 and backbone of Cys172. Such binding profile could not be visualized for Rasagiline since it did not participate in H-bond with Cys172. No attractive interaction energy could be estimated between Ile198 and Rasagiline in B3LYP/ Def2-SVP level of calculation (table 3). However our calculations showed that Rasagiline made slightly tighter contact to Tyr398 with regard to compound 7.

The estimated binding energy for Tyr326 (table 3) might be interpreted considering a $\pi-\pi$ interaction between relatively parallel phenyl rings of compound 7 and Tyr326 (figure 6).

Analysis of binding energies indicated that the interaction between FAD and compound 7 was supported with higher binding energy $(-1.94 \mathrm{kcal} / \mathrm{mol})$ when compared to the similar interaction with Rasagiline $(-0.97 \mathrm{kcal} /$ mol) (table 3). Visualization of binding modes emphasized that extended lipophilic substituents on C6 and C7 positions of coumarin ring might be desirable in providing efficient interactions with FAD cofactor.

Binding energy estimations of compound 7 assigned attractive interaction energy to the Phe168 residue $(-1.07 \mathrm{kcal} / \mathrm{mol})$. This could to some extent indicate a superior activity of meta-methoxy substituted compounds (2 and 7) due to their orientation towards Phe168. Binding cooperation for the interaction of compound 7 and Tyr 188 was found to be $-0.66 \mathrm{kcal} / \mathrm{mol}$ 


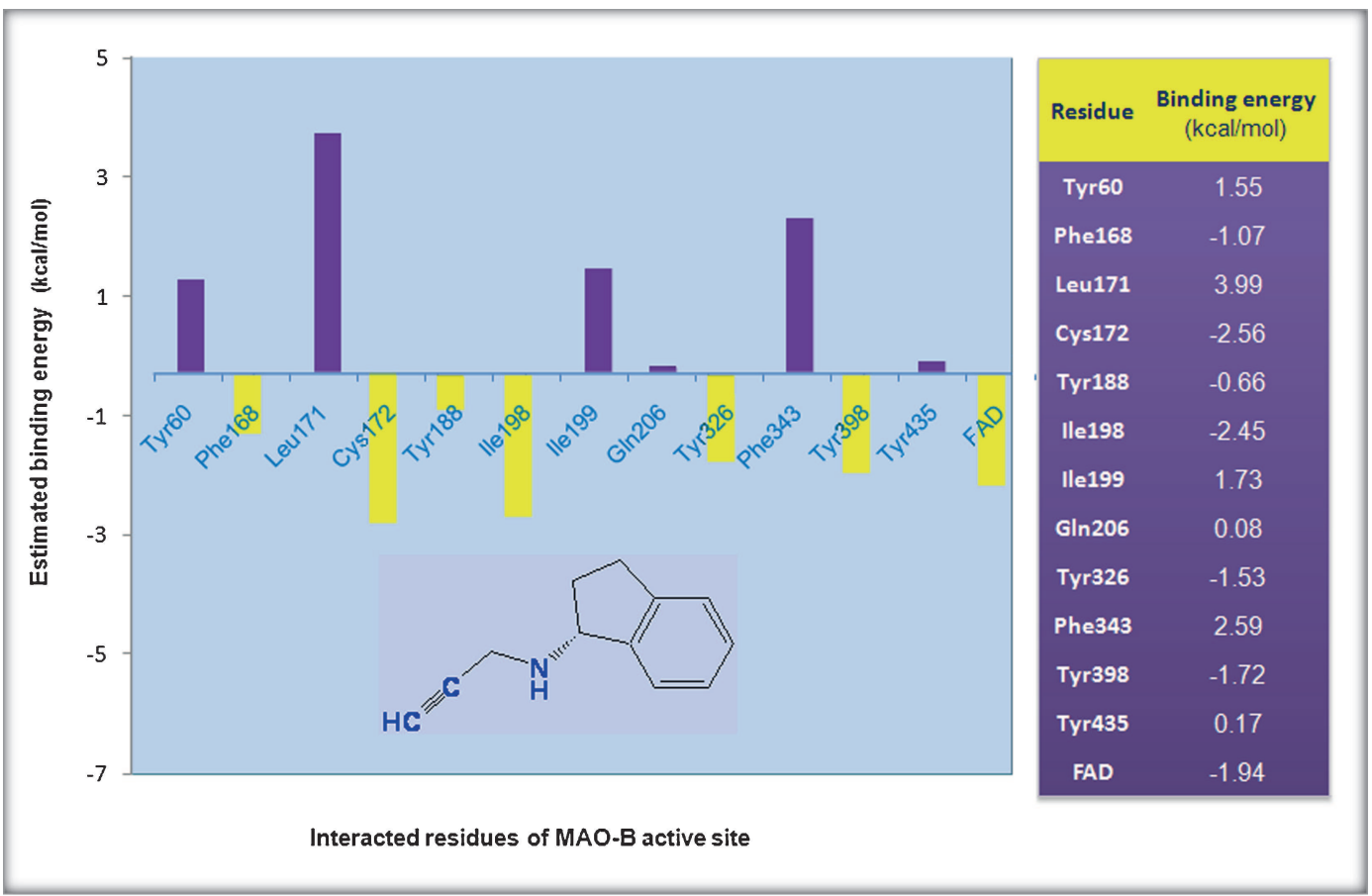

(a)

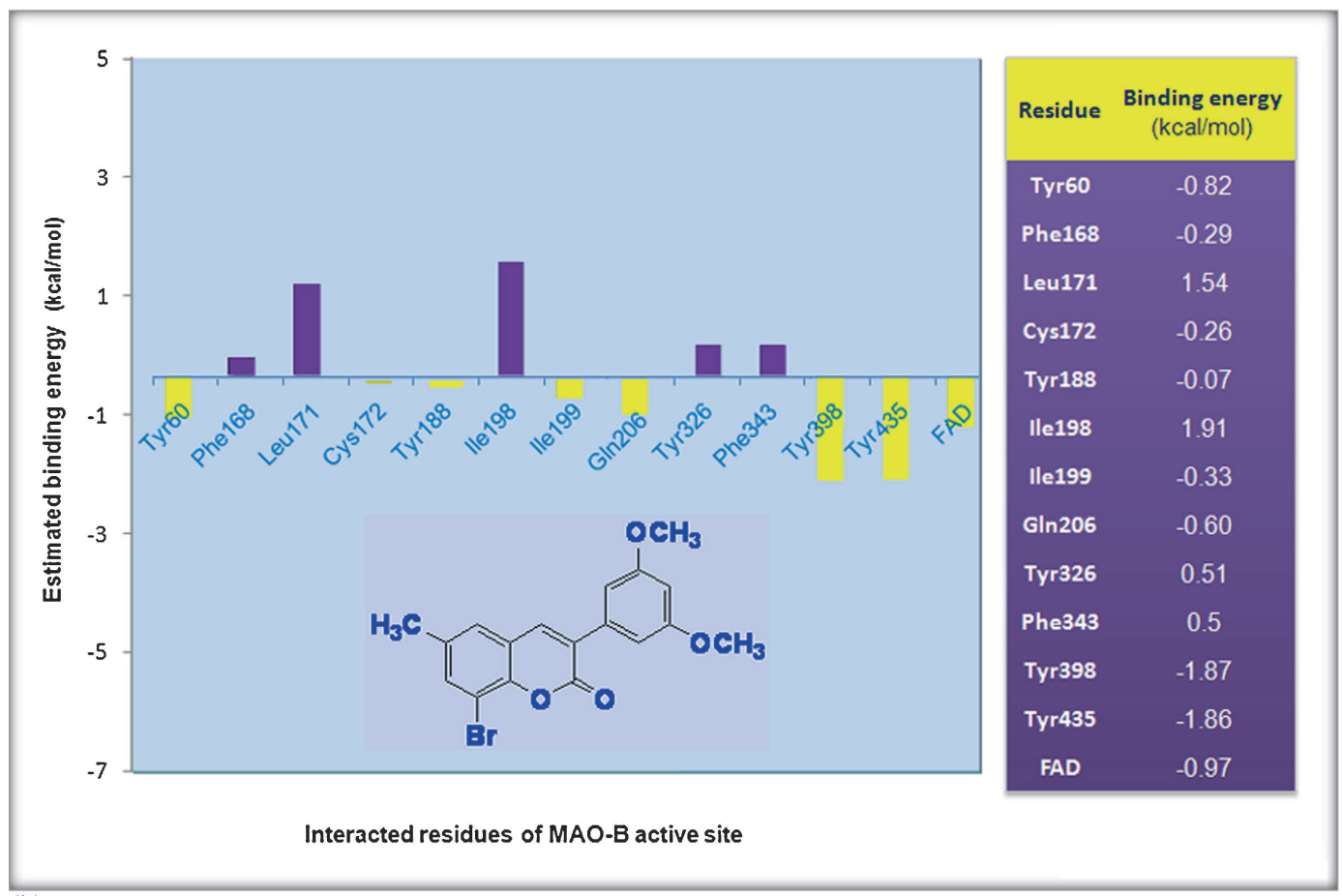

(b)

Figure 5. Binding energies of (a) Rasagiline and (b) compound 7 with individual amino acid residues of MAO-B binding pocket.

at the B3LYP/ Def2-SVP level of calculation. This relatively weak attractive force might be attributed to the bromine substituent on $\mathrm{C} 8$ position of coumarin ring. Regarding the obtained results, it may be proposed that incorporation of extended substituents including aromatic moieties would provide desirable lipophilic and $\pi-\pi$ stacking interactions with Tyr188 and Tyr435.
Major repulsive interactions between compound 7 and MAO-B active site were calculated for Leu171, Phe343 and Ile199 (table 3). These repulsive forces might be the direct outcome of inappropriate geometrical poses with regard to Leu171 and Phe343 and Ile199. According to the obtained results, Leu171 and Phe343 and Ile199 contacting groups of the ligand need to be 
Table 3. Mulliken Partial atomic charges for 8-bromo-3-(3',5'-dimethoxyphenyl)-6 methylcumarin (Compound 7) induced by various residues of MAO-B active site.<smiles>COc1cc(OC)cc(-c2cc3cc(C)cc(Br)c3oc2=O)c1</smiles>

\begin{tabular}{|c|c|c|c|c|c|c|c|c|c|c|c|}
\hline Atom type & Free ligand & Tyr60 & Phe168 & Leu171 & Cys172 & Ile198 & Ile199 & $\mathrm{G} \ln 206$ & Tyr326 & Tyr398 & Tyr435 \\
\hline C1 & $-0.099^{a}$ & 0.133 & 0.131 & 0.131 & 0.131 & 0.135 & 0.131 & 0.131 & 0.131 & 0.131 & 0.131 \\
\hline $\mathrm{C} 2$ & 0.092 & -0.019 & -0.020 & -0.018 & -0.018 & -0.018 & -0.018 & -0.020 & -0.020 & -0.018 & -0.018 \\
\hline C3 & -0.160 & 0.022 & 0.023 & 0.023 & 0.023 & 0.022 & 0.022 & 0.023 & 0.023 & 0.023 & 0.023 \\
\hline $\mathrm{C} 4$ & 0.113 & 0.282 & 0.282 & 0.281 & 0.281 & 0.282 & 0.281 & 0.282 & 0.282 & 0.281 & 0.281 \\
\hline C5 & -0.132 & 0.115 & 0.115 & 0.116 & 0.116 & 0.117 & 0.116 & 0.115 & 0.115 & 0.116 & 0.116 \\
\hline C6 & 0.075 & -0.081 & -0.081 & -0.083 & -0.082 & -0.084 & -0.082 & -0.081 & -0.080 & -0.083 & -0.082 \\
\hline C7 & 0.084 & -0.018 & -0.015 & -0.014 & -0.014 & -0.018 & -0.014 & -0.014 & -0.015 & -0.014 & -0.014 \\
\hline $\mathrm{O} 8$ & -0.136 & -0.499 & -0.499 & -0.499 & -0.499 & -0.498 & -0.499 & -0.499 & -0.499 & -0.499 & -0.499 \\
\hline C9 & 0.400 & 0.550 & 0.549 & 0.550 & 0.549 & 0.550 & 0.550 & 0.550 & 0.550 & 0.550 & 0.550 \\
\hline $\mathrm{C} 10$ & $-\mathbf{0 . 1 2 0}$ & -0.013 & -0.014 & -0.013 & -0.013 & -0.013 & -0.013 & -0.013 & -0.013 & -0.013 & -0.013 \\
\hline $\mathrm{C} 11$ & 0.119 & 0.003 & 0.004 & 0.003 & 0.002 & 0.003 & 0.002 & 0.003 & 0.003 & 0.003 & 0.003 \\
\hline $\mathrm{O} 12$ & -0.125 & -0.465 & -0.466 & -0.466 & -0.465 & -0.466 & -0.466 & -0.465 & -0.466 & -0.465 & -0.466 \\
\hline Br13 & 0.060 & -0.082 & -0.083 & -0.082 & -0.082 & -0.081 & -0.082 & -0.082 & -0.082 & -0.082 & -0.082 \\
\hline C14 & 0.027 & 0.076 & 0.082 & 0.076 & 0.076 & 0.076 & 0.076 & 0.076 & 0.076 & 0.076 & 0.076 \\
\hline $\mathrm{C} 15$ & -0.060 & -0.052 & -0.070 & -0.052 & -0.052 & -0.051 & -0.051 & -0.052 & -0.052 & -0.052 & -0.052 \\
\hline C16 & 0.126 & 0.323 & 0.339 & 0.323 & 0.323 & 0.319 & 0.316 & 0.323 & 0.323 & 0.323 & 0.323 \\
\hline $\mathrm{C} 17$ & -0.127 & -0.048 & -0.092 & -0.048 & -0.049 & -0.046 & -0.045 & -0.048 & -0.048 & -0.048 & -0.048 \\
\hline C18 & 0.132 & 0.302 & 0.336 & 0.302 & 0.302 & 0.302 & 0.303 & 0.302 & 0.302 & 0.302 & 0.302 \\
\hline C19 & -0.008 & 0.002 & -0.017 & 0.001 & 0.002 & 0.001 & 0.002 & 0.002 & 0.002 & 0.002 & 0.002 \\
\hline $\mathrm{O} 20$ & -0.191 & -0.533 & -0.527 & -0.533 & -0.533 & -0.530 & -0.531 & -0.533 & -0.533 & -0.533 & -0.533 \\
\hline C21 & 0.158 & 0.270 & 0.270 & 0.270 & 0.270 & 0.268 & 0.271 & 0.270 & 0.270 & 0.270 & 0.270 \\
\hline $\mathrm{O} 22$ & -0.180 & -0.531 & -0.515 & -0.531 & -0.531 & -0.529 & -0.532 & -0.531 & -0.531 & -0.531 & -0.531 \\
\hline $\mathrm{C} 23$ & 0.155 & 0.262 & 0.268 & 0.262 & 0.262 & 0.259 & 0.262 & 0.262 & 0.262 & 0.262 & 0.262 \\
\hline
\end{tabular}

${ }^{a}$ Atomic charges with hydrogens summed into heavy atoms.

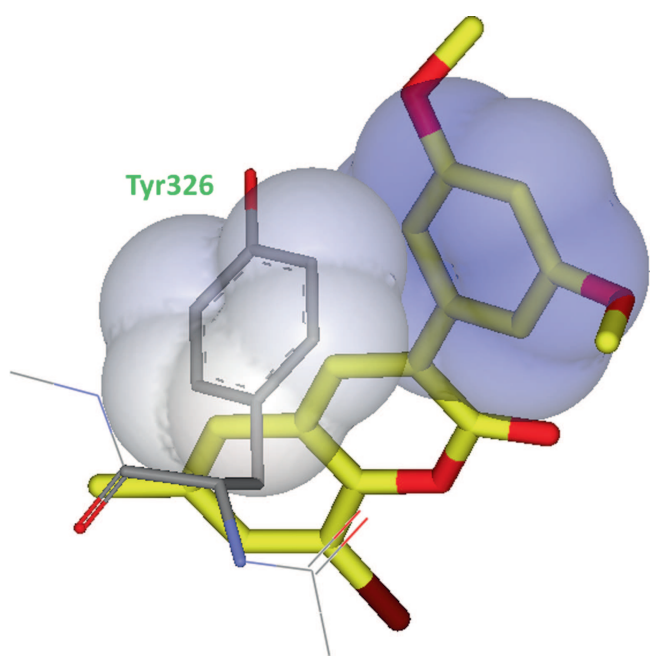

Figure 6. 3D schematic representation of a $\pi-\pi$ interaction between relatively parallel phenyl rings of compound 7 and Tyr326 in the MAO-B binding site. well decorated in future designs of MAO-B blocking 3-phenylcoumarins.

\subsection{Induced polarizability}

Stereoelectronic contributions are very important in ligand-receptor interactions. The binding orientation of the ligand in the active site of a receptor is determined by the complementary fitness between electronic and steric surfaces of ligand and receptor. A part of stereoelectronic effect may be interpreted as ligand polarizability which is induced by different residues of the active site. For this purpose, induced ligand polarizabilities in terms of partial atomic charges for compound 7 were estimated. ${ }^{30}$

Ligand polarizability can be defined as the difference between partial charges in the bound and unbound 
states of a typical enzyme inhibitor. The larger induced polarizability value indicates higher participation of electrostatic interactions in binding of amino acid to the ligand. The relevant induced polarization effects are summarized in table 3. For more clarification, despite the similarity of partial charges in the case of various amino acids, all the data were incorporated.

Careful inspection of the data indicated that large polarization effects occurred at atomic positions attributed to key electrostatic interactions (O8, O12, $\mathrm{O} 20$ and $\mathrm{O} 22$ ) while $\mathrm{C} 15$ and $\mathrm{C} 19$ atoms tolerated the least electrostatic changes (table 3 ) showing their buried orientation at the active site of MAO-B. An important point to note is that hydrophobic forces are also electrostatic in nature since they are caused by correlations in the fluctuating polarizations of nearby particles.

The resultant polarization effects might also be elucidated in terms of induced negative or positive electronic effects. Electronegative atoms O8, O12, Br13, O20 and O22 accept negative induced effect in the binding cavity of MAO-B indicating their contribution to the electrostatic interactions with corresponding amino acids. Other atoms bearing negative induced effect are $\mathrm{C} 2$, C6, $\mathrm{C} 7$ and $\mathrm{C} 11$.

\section{Conclusions}

A series of experimentally tested 3-phenylcoumarin MAO-B inhibitors were elucidated for their intermolecular binding interaction with recombinant human MAO-B enzyme via docking/QM methods. The results were in logical accordance with previous data in the literature. Structure activity relationship (SAR) studies indicated that coumarin ring may be a good substitution pattern for indan ring of Rasagiline in binding to the MAO-B active site. 3-(ortho-methoxyphenyl) substituted coumarins might not be appropriate pharmacophores for MAO-B inhibition while 3-(metamethoxyphenyl) substituted ones can be regarded as suitable choices. Aromatic rings substituted at C3 of coumarin ring might take part in $\pi-\pi$ interactions with Tyr326 via their semi-parallel orientation towards the phenyl ring of Tyr326. Incorporation of 8-halo substituent to the coumarin ring might provide additional electrostatic/lipophilic contact with Tyr188. It seemed that the existence of 6-alkyl moieties afforded a suitable scaffold to make efficient interactions with FAD cofactor of MAO-B binding site. Our results indicated that analysis of intermolecular binding energies between ligand and various constituents of the active site may be a complementary approach to docking simulations in achieving MAO-B inhibitory pharmacophores.
Furthermore, induced polarizabilities of ligand in the active site of the enzyme might give an idea about the exposed or buried parts of the ligand in the MAO-B binding pocket. The outcomes of this study might be useful in developing structure binding relationship of some coumarin derivatives with the aim of achieving potent small molecule MAO-B inhibitors.

\section{Acknowledgements}

This work was financially supported by Research Council of Ardabil University of Medical Sciences.

\section{References}

1. Binda C, Wang J, Pisani L, Caccia C, Carotti A, Salvati P, Edmondson D E and Mattevi A 2007 J. Med. Chem. 505848

2. Tipton K F, Boyce S, O'Sullivan J, Davey G P and Healy J 1965 Curr. Med. Chem. 11

3. Domino E F and Khanna S S 1976 Am. J. Psychiatry 133323

4. Meyer J H, Ginovart N, Boovariwala A, Sagrati S, Hussey D, Garcia A, Young T, Praschak-Rieder N, Wilson A A and Houle S 2006 Arch. Gen. Psychiatry 63 1209

5. Matos M J, Viña D, Picciau C, Orallo F, Santana L and Uriarte E 2009 Bioorg. Med. Chem. Lett. 195053

6. Strolin-Benedetti M and Dostert P 1992 Adv. Drug Res. 2365

7. Yamada M and Yasuhara H 2004 Neurotoxicol 25215

8. Van der Walta E M, Milczek E M, Malan S F, Edmondson D E, Castagnoli N, Bergh J J and Petzer J P 2009 Bioorg. Med. Chem. Lett. 192509

9. Fernandez H H and Chen J J 2007 Pharmacotherapy 27 $174 \mathrm{~S}$

10. Roussaki M, Kontogiorgis C M, Hadjipavlou-Litina D, Hamilakis S and Detsi A 2012 Bioorg. Med. Chem. Lett. 203889

11. Matos M J, Perez-Cruz F, Vasquez-Rodriguez S, Uriarte E, Santana L, Borges F and Olea-Azar C 2013 Bioorgan. Med. Chem. 213900

12. Delogu G L, Serra S, Quezada E, Uriarte E, Vilar S, Tatonetti N P and Viña D 2014 Chem. Med. Chem. 9 1672

13. Delogu G, Picciau C, Ferino G, Quezada E, Podda G, Uriarte E and Viña D 2011 Eur. J. Med. Chem. 461147

14. Viña D, Matos M J, Ferino G, Cadoni E, Laguna R, Borges F, Uriarte E and Santana L 2012 Chem. Med. Chem. 7464

15. Matos M J, Viña D, Janeiro P, Borges F, Santana L and Uriarte E 2010 Bioorg. Med. Chem. Lett. 205157

16. Razzaghi-Asl N, Ebadi A, Edraki N, Shahabipour S and Miri R 2013 Med. Chem. Res. 223259

17. Morris G M, Huey R, Lindstrom W, Sanner M F, Belew R K, Goodsell D S and Olson A J 2009 J. Comput. Chem. 302785

18. Sanner M F 1999 J. Mol. Graphics Model. 1757 
19. Veselovsky A V, Ivanov A S and Medvedev A E 2004 NeuroToxicol. 2537

20. Fogarasi G, Zhou X, Taylor P W and Pulay P $1992 \mathrm{~J}$. Am. Chem. Soc. 1148191

21. Klamt A and Schüürmann G J 1993 Chem. Soc. Perkin Trans. 2799

22. Neese F 2011 ORCA-an ab initio, density functional and semiempirical program package. University of Bonn.

23. Putta S and Beroza P 2007 Curr. Top. Med. Chem. 7 1514

24. Jorgensen W 2004 Science $\mathbf{3 0 3} 1813$

25. Laurie R, Alasdair T and Jackson R M 2006 Curr. Protein Pept. Sci. 7395
26. Sellers R P, Alexander L D, Johnson V A, Lin C C, Savage J, Corral R, Moss J, Slugocki T S, Singh E $\mathrm{K}$ and Davis M R 2010 Bioorg. Med. Chem. Lett. 18 6822

27. Hevener K E, Zhao W, Ball D M, Babaoglu K, Qi J, White S W and Lee R E 2009 J. Chem. Inf. Model. 49 444

28. Toprakc1 M and Yelekc K 2005 Bioorg. Med. Chem. Lett. 154438

29. Razzaghi-Asl N, Hemmateenejad B, Ebadi A, Shahabipour S and Miri R 2014 J. Comput. Methods Sci. Eng. In press

30. Mulliken R S 1955 J. Chem. Phys. 232343 